\title{
Informalidad partidaria y mediaciones entre dirigentes y militantes en México: los casos del PAN, el PRI, el PRD y MORENA*
}

\author{
[Versión en Castellano] \\ Party Informality and Mediations Between Leaders and Militants in \\ Mexico: the Cases of PAN, PRI, the PRD and MORENA \\ Informalidade partidista e mediações entre líderes e militantes no \\ México: os casos do PAN, do PRI, do PRD e da MORENA
}

Recibido el 14 de diciembre, 2018. Aceptado el 26 de febrero, 2020.

Alberto Espejel-Espinoza**
https://orcid.org/0000-0002-9035-5061
México
Mariela Díaz-Sandoval***
https://orcid.org/0000-0002-3900-6202

Para citar este artículo:

Espejel-Espinoza, Alberto;

Díaz-Sandoval, Mariela (2020).

Informalidad partidaria y

mediaciones entre dirigentes

y militantes en México: Ios casos del PAN, el PRI, el PRD y MORENA.

Ánfora, 27(49), 173-196.

https://doi.org/10.30854/anfv27.n49.2020.744

Universidad Autónoma de Manizales. ISSN 0121-6538/

e-ISSN 2248-6941.

CC BY-NC-SA 4.0

\section{Resumen}

Objetivo: el artículo propone evidenciar la lógica informal al interior de los partidos políticos en México, entendida como un mecanismo de mediación entre militantes y dirigentes. Metodología: desde una

\footnotetext{
* Investigación realizada gracias al Programa de Apoyo a la Investigación para el Desarrollo y la Innovación (PAIDI) "La militancia de los partidos políticos en México: democracia interna y procesos de toma de decisiones", auspiciado por la Facultad de Estudios Superiores Acatlán, de la UNAM. Los investigadores declaran que no hubo conflictos de intereses en la ejecución del proyecto de investigación.
}

** Doctor en Ciencia Política por la UNAM. Politólogo. Profesor de la Facultad de Estudios Superiores Acatlán, de la Universidad Autónoma de México. Miembro del Sistema Nacional de Investigadores. Correo electrónico: alberto.espejel.espinoza@gmail.com

*** Doctora en Ciencias Sociales por FLACSO, sede México. Socióloga. Profesora del Instituto Internacional de Estudios Políticos Avanzados de la Universidad Autónoma de Guerreo, Candidata al Sistema Nacional de Investigadores. México. Correo electrónico: mariela.diaz@flacso.edu.mx 
aproximación cualitativa, se exploró el rol de la informalidad en el Partido Revolucionario Institucional (PRI), el Partido Acción Nacional (PAN), el Partido de la Revolución Democrática (PRD), y Movimiento Regeneración Nacional (MORENA). Resultados: se encontró que la informalidad es de vital importancia, ya que, en algunos casos, su ausencia es signo de polarización interna (PAN); en otros, da evidencia de la existencia de acuerdos más allá de lo establecido en los estatutos (PRI). En algunos más, se muestra la realidad fraccionada de la organización (PRD). Conclusiones: la informalidad puede ilustrar el papel discrecional de ciertos liderazgos, así como la adopción de estrategias que contravienen la legislación electoral en aras de mantener ventaja sobre los posibles competidores (MORENA).

Palabras-clave: Política; Partidos políticos mexicanos; Vida política; Informalidad partidaria; Dirigentes y militantes.

\section{Abstract}

Objective: this article aims to demonstrate the informal logistics within the political parties in Mexico. It is understood as a mechanism of mediation between militants and leaders. Methodology: from a qualitative approach, the role of informality in the Institutional Revolutionary Party (PRI), the National Action Party (PAN), the Party of the Democratic Revolution (PRD), and the National Regeneration Movement (MORENA) was explored. Results: informality was found to be of vital importance, in some cases, its absence is a sign of internal polarization (PAN); in other cases, it supports the existence of agreements beyond what is established in the Statutes (PRI). In a few more cases, the fractional reality of the organization (PRD) is shown. Conclusions: informality can illustrate the discretionary role of certain leadership, as well as the implementation of strategies that contravene electoral legislation in order to maintain an advantage over potential competitors (MORENA).

Keywords: Policy; Mexican political parties; Political life; Party informality; Leaders and Militants. 


\section{Resumo}

Objetivo: o artigo propõe demonstrar a lógica informal dentro dos partidos políticos no México, entendida como um mecanismo de mediação entre militantes e líderes. Metodologia: a partir de uma abordagem qualitativa, foi explorado o papel da informalidade no Partido Revolucionário Institucional (PRI), no Partido de Acción Nacional (PAN), no Partido da Revolución Democrática (PRD) eno Movimiento Nacional de Regeneración Nacional (MORENA). Resultados: verificou-se que a informalidadeé de vital importância, uma vez que, em alguns casos, sua ausência é sinal de polarização interna (PAN); em outros, evidencia a existência de acordos além do estabelecido nos estatutos (PRI). Em alguns outros, a realidade fracionária da organização é mostrada (PRD). Conclusões: a informalidade pode ilustrar o papel discricionário de certos líderes, bem como a adoção de estratégias que violam a legislação eleitoral, a fim de manter uma vantagem sobre os concorrentes em potencial (MORENA).

Palavras-chave: Política; Partidos políticos mexicanos; Vida política; Informalidade do partido; Líderes e militantes. 


\section{Introducción}

El estudio de los partidos desde la perspectiva organizativa ha puesto énfasis en la dimensión formal de los procesos internos, lo cual permite dar cuenta de la institucionalización de dichas organizaciones (Panebianco, 1990). Sobre ello existe una larga tradición de trabajos que, para la experiencia mexicana, da cuenta del origen e institucionalización de los partidos (Reveles, 2002, 2003 y 2004). Sin embargo, algunos otros autores plantean la existencia de lógicas informales que son conocidas, acatadas y reproducidas, las cuales generan un clima de certidumbre organizativa, a pesar de no formar parte de los estatutos (Duque, 2005; Randall y Svásand, 2002; Levitsky, 2003; Levitsky y Freidenberg, 2007).

En ese orden de ideas, el objetivo de este trabajo es mostrar evidencia sobre la existencia de la informalidad partidaria, es decir, el conjunto de reglas y prácticas que no forman parte de los estatutos y que, sin embargo, son seguidas, conocidas, aceptadas y reproducidas al interior de las organizaciones partidistas. En este sentido, se pretende aportar a una mejor compresión de las dinámicas informales de los partidos en México, mismas que han tenido un papel relevante en las trayectorias organizativas.

Así, se propone responder a las siguientes preguntas: ¿cuáles son las lógicas informales subyacentes a los principales partidos políticos en México?, y ¿qué papel juegan dichas lógicas en sus características organizativas? Para dar respuesta a tales cuestionamientos, el artículo inicia, en primer lugar, con una discusión general sobre la importancia de la informalidad en los partidos. Enseguida se muestran los aspectos metodológicos que guiaron la realización de la presente investigación. Después, como parte de los resultados, se discute el caso del PRI, sección donde se resaltan las atribuciones presidenciales que tenía el titular del Ejecutivo Federal, tales como palomear y/o remover dirigentes nacionales. Posteriormente, se muestra el caso del PAN, en donde la lógica informal permitió dotar de legitimidad a los procesos de selección de dirigencia o candidatura presidencial. Le sigue el caso del PRD, destacando que la lógica informal ha proporcionado certeza en la toma de decisiones dentro de la organización. Finalmente, como parte de los resultados, se clarifica el caso de MORENA, destacando el nombramiento de los Promotores de la Soberanía Nacional, por parte del líder moral del partido. De hecho, tales nombramientos son otorgados a quienes se convertirán en los candidatos a puestos de elección popular. Finalmente, se concluye con una discusión respecto a los alcances de la lógica informal en los partidos mexicanos, así como las líneas de investigación pendientes en este campo de estudio.

Así pues, de acuerdo con Araujo (2009a), América Latina es una región que aporta evidencia sobre una histórica y conflictiva relación con la ley, la cual ha 
generado interpretaciones peyorativas sobre estas sociedades. Así, el tema de las normas sólo ha sido abordado, remitiendo a la deficiencia de las mismas y, con ello, ha prevalecido la idea de que en la región las sociedades están determinadas por una cultura de la transgresión vía la duplicidad moral.

Sin embargo, derivado de los procesos de transición a la democracia en la región, así como por la emergencia de investigaciones que analizaron la relación con las normas desde una perspectiva fáctica, ha sido relativamente reciente el interés sobre el estudio de las normas. Al respecto, resaltan dos grandes líneas de investigación que comparten la intención de que prevalezca la comprensión y explicación del papel de la norma sobre la condena moral.

En primera instancia, la línea denominada culturas de la transgresión, la cual abandona "la condena cultural unívoca” y, en cambio, hace suya una vocación comprensiva de interpretación sociocultural y política, gracias a lo cual indaga sobre los mecanismos concretos por los que las culturas de la transgresión se perpetúan, renuevan y reproducen. En ese sentido, se estudian, desde tradiciones institucionales, prácticas administrativas, hasta interacciones cotidianas toleradas. Este es el caso de Girola (2009), quien indaga la pluralidad de factores sociales, políticos y económicos que sustentan las culturas de la transgresión en México. Su trabajo muestra una relación particular con las normas, a través de la idea de la cultura del "como si", la cual denota distintas facetas del problema normativo en México, tales como: "la pérdida de sentido normativo, la descomposición del sistema de valores, la tolerancia a la transgresión, la no vigencia de reglas" (Girola, 2009, p. 22). De ahí que, en política "hacemos como si estuviéramos convencidos del valor de la democracia, cuando en realidad muchas veces quisiéramos que otros tomen las decisiones" (Girola, 2009, pp. 47 -48). La autora concluye que las disfunciones de las normas formales se pueden ver como la génesis de órdenes normativos subyacentes, los cuales son una respuesta a la falencia y no vigencia de los órdenes ideales que, pese a todo, operan como modelos.

Una segunda línea de investigación se centra en las normas informales en contextos de interacción. En ese sentido, apartando el juicio moral, se enfoca en los elementos cognitivos, emotivos, políticos y sociales que acompañan el uso cotidiano de las reglas, poniendo énfasis en el estudio de las maneras efectivas por las que los actores movilizan y enfrentan las reglas. Estos trabajos rechazan la idea simplista sobre el bien y el mal, ya sea porque se transgrede la ley en nombre de la moral, porque se hace cumplir la norma vía la transgresión o porque se configuran usos alternativos. En esta línea argumentativa destaca el trabajo de Araujo (2009b) quien se enfoca en el estudio de los sectores con menores recursos en Chile para proponer que los ideales sociales y las experiencias del individuo configuran un tipo de sujeto específico que se relaciona con otros y con las instituciones. Por ello, describe la experiencia social de "pasar a llevar" 
la cual posee dos núcleos centrales: por un lado, la discriminación, ya que revela la invisibilidad para el otro (del que tiene más recursos); por otro lado, refiere a que se es objeto de la violencia y el abuso de poder. Con ello, el ideal normativo se debilita, pues es evidente el no funcionamiento del principio de igualdad, y que la ley, así como el derecho no tienen el potencial para regular las relaciones de los individuos, y de ellos con las instituciones.

Lo anterior da lugar a cuatro tipos de configuraciones de sujeto: 1) el sujeto indignado que reclama por el funcionamiento efectivo de una instancia reguladora de la sociedad; 2) el individuo pragmático que se somete a la norma como una estrategia de inclusión y mantenimiento en el sistema; 3) el sujeto desencantado que, vía la impotencia y la derrota, es un actor pasivo y resignado; 4) el individuo anti sistémico que cuenta con dos modalidades, la primera que, dada la inoperancia del ideal, toma la ley en sus manos, mientras que el segundo abandona el campo de juego y produce otra esfera en la que no puede ser tocado ni por la norma, ni por los otros.

En este sentido, la informalidad vuelve a emerger como pregunta de investigación pertinente en las ciencias sociales, alejada de la condena moral. Al respecto, los partidos políticos latinoamericanos también comenzaron a repensarse, incorporando la dimensión de las lógicas informales, como se muestra a continuación.

La tradición organizativa enfocada en el estudio de los partidos ha mostrado avances importantes, desde su génesis con los trabajos de Michels (1983) y Ostrogorski (2008). Sin duda, de la concepción de que los partidos son organizaciones en las que el militante se subordina al dirigente y éste a la organización, se han dado saltos cualitativos al entender a los partidos como entes complejos que tienden a desarrollar características organizativas más o menos estables. Lo anterior es producto de su historia y dinámica internas, así como de factores externos como el marco institucional en el que se desenvuelven y la competencia política. Al mismo tiempo, se trata de organizaciones que logran, en mayor o menor medida, afianzar vínculos con la población. Ambos aspectos, tanto los organizacionales, como el enraizamiento, suelen englobarse en el concepto de institucionalización ${ }^{1}$.

Uno de los autores más utilizados para entender los niveles de institucionalización de los partidos políticos es Angelo Panebianco, quien tuvo el acierto de proporcionar un esquema de análisis atractivo y útil para medir dicho concepto. En el esquema del teórico italiano, dependiendo de los componentes, hay partidos que parecen condenados al fracaso. Por ejemplo, las organizaciones partidistas que incluyen entre sus ingredientes originarios al carisma y la fraccionalización, aspectos casi fatales para la organización, los condena a la configuración de una débil

1. Para una revisión puntual de las diferentes formas de abordar y medir la institucionalización, así como críticas a las aportaciones formales clásicas véase Duque (2005) y Martínez (2005). 
institucionalización. No obstante, existen partidos que, aún con la presencia de ambos ingredientes, han logrado estabilidad a lo largo del tiempo, como ocurrió con el PRD y el PRI. En el caso del primero, nació de una multiplicidad de grupos que se unieron por el carisma de Cuauhtémoc Cárdenas; en cuanto al segundo, contó con el poder presidencial que interfirió y definió el destino del partido.

Lo anterior invita a repensar un aspecto crucial, pues, si existen partidos con componentes dotados de informalidad y que, pese a ello, sobreviven, es necesario repensar la rutinización como un componente que existe más allá de las vías formales. Para Panebianco (1990), la institucionalización equivale a la consolidación organizativa por vías formales ${ }^{2}$.

Expuesto lo anterior, es preciso utilizar otro tipo de literatura que, si bien reconoce los aportes clásicos de autores como Panebianco (1990) o Huntington (1968), permita dar cuenta de la informalidad en el ámbito partidario latinoamericano en general, y mexicano en particular. Al respecto, existen trabajos de corte teórico y empírico que conviene retomar. En primera instancia, el trabajo de Levitsky (2003) distingue dos conceptos de relevancia para comprender el papel de las reglas informales en los partidos políticos en América Latina. Por un lado, se encuentra lo que denomina infusión de valor, definido como un atributo que la organización adquiere cuando el mantenimiento de la organización se convierte en una meta. Por otro lado, está la rutinización, que se refiere al proceso por el cual las reglas son conocidas, aceptadas y seguidas, sean formales o informales (Levitsky, 2003, p. 254). Por consiguiente, puede presentarse la rutinización por vías informales, acompañada de la infusión de valor, dando lugar a una institucionalización diferente a la planteada por Panebianco.

De ahí que cabe la posibilidad, dada su pertinencia, de reconocer a la institucionalización como algo más que el estricto ajuste a reglas formales. Lo anterior permite dar un justo reconocimiento a la realidad de Latinoamérica y, particularmente, al aporte que O’Donell (1997) hizo hace más de veinte años con "la otra institucionalización”, entendida como el entramado de pautas regularizadas conocidas, practicadas y aceptadas que guían la interacción humana.

Para precisar, Helmke y Levitsky (2003) sostienen que el término institución informal debe ser diferenciado de cultura, redes personales, clientelismo, corrupción, clan y mafia, entre otros.

Por lo tanto, es prudente entenderlas como reglas compartidas, no escritas, creadas, comunicadas e implementadas por fuera de los canales oficiales. En este tenor, los partidos pueden contar con un cúmulo de instituciones informales, lo

2. Algo similar ocurre en el caso de Huntington (1968) quien, pese a referirse a la institucionalización del sistema de partidos, al igual que Panebianco, su definición pone énfasis en las reglas formales. De ahí que Martínez (2005) sostiene que en algunos casos resulta "imposible de acreditar los parámetros de una institucionalización rigurosamente formal" (p. 139). 
cual no significa que éstos no estén institucionalizados. Más bien se trata de una institucionalización diferente que gracias a la informalidad consigue certeza en sus procesos internos. Incluso, el partido puede ceñirse en mayor medida a las reglas formales, pero ello no significa que dejarán de presentarse algunas prácticas informales que cumplen una función concreta.

Por ende, autores como Ortiz Loaiza (2008) se enfocan en detallar, por un lado, las prácticas partidarias en torno a las actividades del partido, ya sea en época electoral o no, en el sistema de partidos en Guatemala. También se concentran en identificar las contradicciones entre dichas prácticas y lo que establece el marco estatutario; así como la identificación de prácticas y actividades no reguladas o inexistentes en el marco legal, que el partido realiza de acuerdo con sus propias costumbres y valores.

Levitsky (2003), por su parte, analiza el caso del Partido Justicialista de Argentina, cuyo origen está marcado por el carisma de su líder, aunque sobrevivió a la muerte de éste. En cuanto a la rutinización, no es un partido institucionalizado formalmente, debido a que su líder frenó, en diversos momentos, la instauración de reglas formales. A lo anterior se agrega la proscripción del partido durante un largo periodo. Respecto a la infusión de valores, la organización es valorada por sus miembros y dirigentes quienes se han comprometido con la sobrevivencia del partido.

Mientras que Pedrosa (2005) realizó una investigación sobre las redes e instituciones informales en el partido Unión Cívica Radical en Argentina. El autor encuentra que el entramado de redes personales, vínculos, comportamientos intercambios de recursos materiales y simbólicos dan vida al partido y permiten lograr sus metas. Su trabajo brinda evidencia sobre la existencia de un gran número de grupos informales flexibles, comandados normalmente por un caudillo que se incrustan en los comités del partido. Lo anterior da como resultado una mezcla de formalidad e informalidad que brinda vitalidad y permanencia al partido.

Por último, en el caso mexicano, Martínez (2005) muestra cómo las fracciones y el carisma pueden acrecentar el proceso de institucionalización, tal como sucedió en el caso del PRD, ya que se constituyen como "vehículos de una institucionalización menos formal, pero existente y efectiva” (Martínez, 2005, p. 367). Esto dotó de cierta estabilidad sui generis a la organización gracias a las prácticas informales para seleccionar dirigencias.

Ahora bien, tomando en cuenta que el objetivo del trabajo es mostrar evidencia sobre la existencia de lo que se denomina aquí informalidad partidaria, a continuación se explica las principales coordenadas teóricas-metodológicas implementadas. En primera instancia, es necesario clarificar la selección de casos. Se optó por indagar a los principales partidos en México, según los votos y 
cargos que obtuvieron en la última elección federal llevada a cabo en 2018. Las organizaciones seleccionadas son el PRI, el PAN, el PRD y MORENA. En el caso de las tres primeras organizaciones, es preciso indicar que se trata de los principales partidos, desde 1989, conformando un sistema de partidos pluralista moderado. En PRI fue el partido hegemónico en México durante largo tiempo (desde 1946, hasta la década de 1990, momento en que comenzó a perder espacios de poder relevantes como gubernaturas, municipios y escaños en el legislativo). Ante este hecho, el PAN ganó la Presidencia de la República en 2000 y 2006. Por su parte, el PRD terminó en segundo lugar en 2006 y 2012. En cuanto a MORENA, esta organización se formó después del éxodo de Andrés Manuel López Obrador del PRD, logrando configurarse como el partido de nueva creación con mayor éxito electoral en México ${ }^{3}$.

Retomando la tipología generada por Helmke y Levitsky (2003, pp. 12-16), se concibe la posible existencia de instituciones informales: 1) complementarias, ya que llenan los vacíos de las reglas formales, sin violarlas, mejorando su desempeño; 2) complacientes, pues violan el espíritu, pero no la letra de la regla formal, ayudando a conciliar intereses divergentes; 3) competidoras, ya que coexisten con reglas formales ineficaces y al seguir una regla (formal o informal) se viola la otra (son reglas particularistas); 4) sustitutivas, pues son creadas o empleadas para alcanzar los resultados que no fueron posibles mediante las reglas formales.

A partir de lo anterior, la intención fue evidenciar qué tipo de lógica informal ha existido al interior de los principales partidos políticos en México.

\section{Metodología}

Teniendo en cuenta que el fin del trabajo fue dar cuenta de la lógica informal, aspecto históricamente relevante en los derroteros organizativos, se optó por el método cualitativo para la recolección y análisis de la información. Así, se usaron documentos oficiales de los partidos, hemerográfica, así como algunas entrevistas en profundidad, con el objetivo de triangular datos. En tanto que, la selección de dicha información se realizó bajo un muestreo de conveniencia (Patton, 2002). La intención, al realizar el análisis de datos, fue "ver si todos los hechos que son de alguna manera relevantes para el marco propuesto se ajustan

3. En ese sentido, en la última elección federal de 2018, obtuvieron los siguientes porcentajes. En el caso de la elección presidencial, MORENA, junto a los partidos (minoritarios) Del Trabajo y Encuentro Social, lograron el 54.74\% de la votación; el PRI de la mano de sus aliados minoritarios, Partido Verde Ecologista de México y Partido Nueva Alianza, obtuvieron el 16.89\% El el PAN, el PRD y el minoritario Movimiento Ciudadano lograron el 22.92\%. En el Senado la votación fue: 45.46, 23.74 y 28.74\%, respectivamente, mientras que en la Cámara de Diputados el porcentaje fue de 45.33, 24.86 y 28.81\%, respectivamente. 
a una única interpretación o la apoyan” (Ragin, 2007, p. 175). Es decir, si las instituciones informales presentes en los cuatro casos estudiados apoyan alguna de las posibilidades o conceptos guía enunciados líneas arriba.

\section{Resultados}

\section{a) El poder presidencial sobre el Revolucionario Institucional}

El PRI surgió en 1946; sus antecedentes inmediatos son el Partido Revolucionario Mexicano (1938) y el Partido Nacional Revolucionario (1929). En los tres, el peso del Presidente de la República fue abismal por encima de los demás caudillos (PNR), los sectores (PRM) y la militancia (PRI), lo cual discurrió vía cauces informales.

En ese sentido, la informalidad fue uno de los resortes del presidencialismo, dado que la atribución de designar a su sucesor, así como remover y designar a dirigentes nacionales y gobernadores no se encontraba inscrita en estatuto alguno. Dicha prerrogativa fue central del periodo que va de 1946 a la década de 1990, ya que en esa década se complicó la relación entre nivel subnacional y nacional derivado del avance de la oposición y la aceptación de derrotas de parte del Presidente. No obstante, el PRI tuvo un largo tiempo de informalidad y ésta fue determinante para sostener la pirámide encabezada por el Presidente.

Si bien, la atribución del "dedazo", referida a la selección del sucesor presidencial (Carpizo, 1987), así como la selección y remoción de gobernadores (Hernández, 2008) han sido estudiadas, no ha ocurrido así con la selección y remoción de dirigentes nacionales.

Respecto al asunto de la selección de dirigentes, la historia del PRI muestra que dicha decisión se ha tomado de acuerdo con los intereses del Presidente, "o a las circunstancias políticas del momento” (Corona, 2003, p. 164). Una primera lectura de la Tabla 1, que abarca la totalidad de dirigentes del PRI de 1946 y hasta antes de perder la presidencia la República, da cuenta de 26 dirigentes nacionales. Un número récord si se tiene en cuenta que estatutariamente cada dirigente debió durar tres años en el encargo. 
Tabla 1. Dirigentes nacionales del PRI (1946-1999)

\begin{tabular}{|c|c|c|}
\hline Periodo & Dirigente nacional & Presidente de la República \\
\hline 1946 & Dr. Rafael Pascasio Gamboa & Manuel Ávila Camacho \\
\hline 1946-1952 & Gral. Rodolfo Sánchez Taboada & Miguel Alemán Valdés \\
\hline $1952-1956$ & Gral. Gabriel Leyva Velázquez & \multirow{2}{*}{ Adolfo Ruíz Cortines } \\
\hline 1956-1958 & Gral. Agustín Olachea Avilés & \\
\hline 1958-1964 & Gral. Alfonso Corona del Rosal & Adolfo López Mateos \\
\hline 1964-1965 & $\begin{array}{c}\text { Lic. Carlos Alberto Madrazo } \\
\text { Becerra }\end{array}$ & \multirow{3}{*}{ Gustavo Díaz Ordaz } \\
\hline 1965-1968 & Dr. Lauro Ortega Martínez & \\
\hline $1968-1970$ & C. Alfonso Martínez Domínguez & \\
\hline 1970-1972 & Prof. Manuel Sánchez Vite & \multirow{3}{*}{ Luis Echeverría Álvarez } \\
\hline $1972-1975$ & Lic. Jesús Reyes Heroles & \\
\hline $1975-1976$ & Li. Porfirio Muñoz Ledo & \\
\hline 1976-1979 & Lic. Carlos Sansores Pérez & \multirow{4}{*}{ José López Portillo } \\
\hline 1979-1981 & Lic. Gustavo Carvajal Moreno & \\
\hline 1981 & C. Javier García Paniagua & \\
\hline 1981-1982 & Lic. Pedro Ojeda Paullada & \\
\hline 1982-1986 & Lic. Adolfo Lugo verduzco & \multirow{2}{*}{ Miguel de la Madrid Hurtado } \\
\hline 1986-1988 & Lic. Jorge de la Vega Domínguez & \\
\hline 1988-1992 & Lic. Luis Donaldo Colosio & \multirow{5}{*}{ Carlos Salinas de Gortari } \\
\hline 1992 & Rafael Rodríguez Barrera & \\
\hline 1992-1993 & Genaro Borrego Estrada & \\
\hline 1993-1994 & Fernando Ortiz Arana & \\
\hline 1994 & Ignacio Pichardo Pagaza & \\
\hline 1994-1995 & María de los Ángeles Moreno & \multirow{4}{*}{ Ernesto Zedillo Ponce de León } \\
\hline 1995-1996 & Santiago Oñate Laborde & \\
\hline 1996-1997 & Humberto Roque Villanueva & \\
\hline 1997-1999 & Mariano Palacios Alcocer & \\
\hline
\end{tabular}

Fuente: elaboración propia con base en PRI (2003)

De igual forma, la coyuntura política ha prevalecido sobre cualquier otro criterio para designar dirigente. Aunado a ello, dicha decisión ha recaído exclusivamente en el Presidente en turno. Así, Gustavo Díaz Ordaz y Luis Echeve- 
rría contaron con tres dirigentes en su sexenio, lo cual se acrecentó a cuatro en el período de José López Portillo. En tanto que, en la medida que la oposición obtuvo triunfos a nivel sub nacional, el número dirigentes aumentó. De ahí que, Carlos Salinas contó con cinco y Ernesto Zedillo con cuatro. En todos los casos el designado contó con la venia del Presidente y llegó al cargo sin competencia. De hecho, las asambleas (para elección ordinaria) o consejos nacionales (para extraordinaria) para elegir a los dirigentes no fueron más que instancias de legitimación de una decisión previamente tomada por el Presidente.

Espejel-Espinoza (2016) apunta que, en el caso de algunos dirigentes, atendiendo los intereses presidenciales en turno, se trató de designaciones que tenían por objetivo dirigir al partido durante un proceso electoral sucesorio. Por consiguiente, una vez culminado el proceso, los dirigentes fueron premiados con alguna secretaría de Estado. Por ejemplo, a Porfirio Muñoz, una vez que José López Portillo fue designado Presidente, le fue encargada la Secretaria de Educación Pública.

Por lo anterior, otros dirigentes llegaron para conducir al partido durante el sexenio iniciado. Por ejemplo, Rodolfo Sánchez de 1946 a 1950, Alfonso Corona del Rosal de 1958 a 1964, Jesús Reyes Heroles de 1972 a 1975, Adolfo Lugo de 1982 a 1986 y Luis Donaldo Colosio de 1988 a 1992, entre otros. De ahí que, el gran elector de los dirigentes del PRI en su historia previa al año 2000 fue el Presidente en turno.

En cuanto a la remoción, en el mismo lapso, solo el Presidente pudo remover dirigentes. Además, dicha remoción aconteció por causes informales, pues los estatutos no planteaban tal atribución. Como ya se apuntó, algunos dirigentes fueron removidos por el recién llegado a la Presidencia, como premio a su lealtad durante la campaña presidencial. Sin embargo, en otros casos la remoción aconteció debido a que el dirigente trastocó los acuerdos informales entre el Presidente y el partido o cuando no lograban conservar la estabilidad en la organización.

Un ejemplo del primer caso fue lo acontecido a Genaro Borrego en el sexenio de Carlos Salinas, al intentar brindar cierta autonomía al partido, fue removido. Similar fue el caso de Carlos Sansores en 1979, debido a los problemas que tuvo con Jesús Reyes Heroles, entonces Secretario de Gobernación (Partido Revolucionario Institucional [PRI], 2003, p. 194).

En el segundo tipo de remoción, fue el caso de Adolfo Lugo Verduzco que fue removido ante la imposibilidad de apaciguar a la Corriente Democrática a finales de los ochenta (PRI, 2003, p. 213). De ahí que, el depositario del control político de los dirigentes, previo al año 2000, fue el Presidente, ya sea premiando la lealtad o castigando la desobediencia o nula efectividad.

Así, el PRI es un partido que presentó un fuerte contenido de aspectos informales, es decir, reglas alejadas de los estatutos que se conocen, siguen, acep- 
tan y ayudaron a dar certeza a los actores internos. Siguiendo la clasificación de Helmke y Levitsky (2003) el PRI proporciona evidencia de una lógica complementaria, pues el cumplimiento de la regla informal (nombrar y remover dirigentes de parte del Presidente en turno) supone la violación de la regla formal ineficaz (nombrar dirigentes a través de los congresistas). Al mismo tiempo, daban evidencia de la enorme concentración de poder en manos del Presidente en turno, pues era el "gran elector" de los dirigentes, así como el único capaz de ejercer control político hacia éstos, de 1946 hasta antes del año 2000.

Con la pérdida de las Presidencia la República, el PRI transitó a un estado de orfandad, situación que permitió la emergencia de otros centros de poder como los gobernadores, los líderes parlamentarios y los dirigentes. Con ello, el control se redujo; sin embargo, aunque ello no supuso la erradicación de herencias autoritarias, en 2012, cuando el PRI logró ganar la Presidencia, Pedro Joaquín Coldwell fue premiado por su lealtad al pasar de dirigente del partido a Secretario de Energía, bajo el gobierno de Enrique Peña Nieto.

\section{b) La cortesia "informal" en Acción Nacional}

En 1939 surgió el también llamado partido blanquiazul, organización que ha dado muestras de una enorme institucionalidad formal a lo largo de su historia; sin embargo, poco se ha explorado la informalidad que ha pervivido desde su génesis. Basta recordar que, en sus inicios, Manuel Gómez Morín estuvo al frente de la dirigencia por 10 años, a pesar de contravenir los estatutos. Si bien se trató de una decisión justificada por las deficiencias organizativas y lo azaroso del momento originario (González y Gómez, 2010), no deja de ser significativa la presencia de la informalidad en su origen, sobre todo en un partido que surge como respuesta al predominio de líderes y caudillos sobre las instituciones y que, por ende, otorgó gran importancia a las reglas formales. En ese sentido, la informalidad en el PAN volvió a presentarse en acontecimientos de primer orden como los procesos de selección de dirigentes y candidatos presidenciales. En ellos, se configuró una regla, la cortesía informal, la cual implicó que el segundo lugar declinaría en sus aspiraciones para que el primer lugar alcanzara el porcentaje requerido. Esta regla no escrita permitió la celebración de competencias cordiales por la selección de dirigente.

Así, la declinación de un candidato a favor de otro se presentó en diversos momentos de la vida de Acción Nacional, mostrando que la competencia discurría por cauces normales. Tal fue el caso de la XIII Convención Nacional de 1957, momento en el que José González Torres decidió cederle sus votos a Luis H. Álvarez en la segunda votación, para que éste lograra el 80\% requerido para ser candidato presidencial. En 1970, Salvador Rosas Magallón retiró su candida- 
tura después de una segunda ronda de votación, permitiendo el triunfo de Efraín González Morfín. Dicha tendencia a declinar se presentó en la mayoría de casos hasta que, en 1999, el PAN decidió seleccionar a su candidato presidencial a través de la decisión de la militancia.

No obstante, en coyunturas donde no se declinó, la polarización se hizo presente. Por ejemplo, en las convenciones nacionales de 1975 y 1976, ningún contendiente retiró su candidatura, provocando que el PAN se quedara sin candidato presidencial, dado que ningún contendiente alcanzó el 80\% necesario. Así, ante la polarización, se rompió la regla informal de que el segundo lugar retiraba su candidatura.

En cuanto a la selección de dirigentes nacionales, dicha tendencia se repitió. En los casos en donde la polarización no emergió, el candidato posicionado en el segundo lugar declinó a favor del primero. No obstante, hubo ocasiones en que la polarización no permitió la declinación (como en 1990), lo cual fue el preludio de la crisis por venir (como ocurrió en 1975).

Ejemplos de declinaciones se presentaron en 1987, cuando Pablo Emilio Madero, luego de dos votaciones, declinó a favor de Luis H. Álvarez, quien logró el 66\% que solicitaban los estatutos. En 1996, después de la primera ronda, Ernesto Ruffo declinó a favor de Felipe Calderón. En 2002, Carlos Medina Plascencia declinó en favor de Luis Felipe Bravo, permitiendo el triunfo del grupo que marcó un distanciamiento del Vicente Fox. No obstante, en 2005, Carlos Medina Plascencia declinó en la segunda ronda a favor de Manuel Espino, dirigente afín a Vicente Fox; mientras que, en 2010 Roberto Gil Zuarth declinó ir a una segunda ronda, hecho que permitió el triunfo de Gustavo Madero. Con lo anterior se consumó la derrota del candidato afín a Felipe Calderón, entonces Presidente de México.

Ahora bien, han existido casos en que dicha práctica informal (la declinación) no se ha presentado, dando muestra de conflictos al interior del partido. En 1990, la dirigencia fue disputada por Luis H. Álvarez y Gabriel Jiménez Remus; coyuntura en la que se requirieron cuatro rondas de votación. Llama la atención que Jiménez no retiró su votación, lo que mostró un claro síntoma de conflicto al interior del PAN, mismo que derivó que, en 1992, se conformara el Foro Democrático y Doctrinario de Acción Nacional. Dicho Foro representó la escisión más importante del PAN, pues, entre quienes abandonaron el partido se encontraban ex dirigentes y ex candidatos presidenciales (José González Torres y Pablo Emilio Madero), así como ex secretarios del partido (Bernardo Bátiz y Jesús González).

Por consiguiente, si bien el PAN es un partido en el que la institucionalidad formal es fundamental, la organización no ha quedado exento de prácticas informales en momentos importantes, como ha ocurrido en la selección de can- 
didatos presidenciales y de dirigentes nacionales. En coincidencia con Helmke y Levitsky (2003), el PAN brinda evidencia sobre la existencia de una lógica complementaria, ya que la cortesía de declinar por el primer lugar permitió llevar a buen puerto los procesos de selección de dirigentes, sin desgastar al partido. Así, cuando no se presentó la regla informal, la polarización del partido quedó de manifiesto, llegando a casos extremos como en 1976, cuando el PAN no pudo designar candidato presidencial, o en 1990, cuando en el proceso de selección de dirigente nacional nadie declinó. En ambos casos, el incumplimiento de la regla informal fue signo de las salidas y crisis que acontecieron más adelante, como ocurrió, en 1978, con los efrainistas y, en 1992 con los foristas.

\section{c) El "modus vivendi" de la Revolución, ¿democrática?}

El PRD, o partido del Sol Azteca, es un caso sui generis en la conformación partidista en México, pues su origen presentó dos ingredientes poco valorados por la literatura partidista: el líder carismático y los grupos previamente organizados. Lo anterior permitió que el partido tuviera una peculiar articulación, pues de 1989 a 1999, predominó el líder carismático. No obstante, a partir del año 2000, en la organización emergió una pugna entre grupos consolidados y el líder carismático, dando lugar a una etapa en la que aquellos son quienes toman las principales decisiones y, además, se sirven instrumentalmente de los líderes carismáticos (Espejel-Espinoza, 2016).

En ese orden de ideas, la vida diaria del PRD ha estado permeada por la informalidad, entendida como un mecanismo generador de certezas entre la coalición dominante, específicamente en temas como la selección de dirigentes, en los criterios de la elección y en las acciones que deben llevarse a cabo en ausencia del titular de la dirigencia. A su vez, la informalidad le alerta a la militancia y a cuadros intermedios sobre sus posibilidades de incidencia al interior del partido (en tanto pertenezca a un grupo) y en torno al funcionamiento real del partido (en procesos internos).

En cuanto a la certeza que otorga a la coalición dominante, el PRD se ha caracterizado por ser un partido donde el control político se deposita en los grupos que lo conforman. En ese sentido, la práctica informal conocida como "quitapón" refiere que, ante la ausencia de un integrante de la dirigencia, por renuncia o porque su grupo político lo retiró del cargo, corresponde al mismo grupo político colocar al sucesor. Lo anterior, dista mucho de lo que marcan los estatutos. Así, "cuando por alguna razón alguien se va de candidato, alguien renuncia, alguien tiene un problema o hay un debate o discrepancias, y se tiene que nombrar un sustituto, ese sustituto viene de la corriente que lo puso" (Saúl 
Escobar, entrevista con el autor, 08 de octubre de 2011). Dicha práctica ha emergido en contextos nacionales y subnacionales (Espejel-Espinoza, 2010).

Respecto a la certeza en los cuadros medios y la militancia, existen dos frases que ejemplifican el peso de los grupos sobre el reclutamiento y sobre las posibilidades de que actores ajenos a los sectores dirigentes pertenezcan a la coalición dominante. En primer lugar, la frase “te alineas o quedas fuera” refiere a que en las reuniones del partido, en las que se tomarán decisiones, el militante "de a pie" tiene poco qué hacer, pues, para incidir en un partido tan fraccionado, debe alinearse a un grupo para obtener algún incentivo material o selectivo. En ese sentido; "O perteneces a alguna corriente política dentro del partido o no aspiras... Dentro del PRD tú vienes, te afilias y como afiliado no eres nada... tú vales por los votos que traigas, si tú eres tú solo, no vales nada... [sic]” (Netzahualcóyotl González, entrevista con el autor, 20 de mayo de 2005).

Finalmente, en cuanto a la certeza sobre el funcionamiento real del partido, la frase "acuerdo mata estatuto", de acuñación perredista, es una de las más conocidas en el argot político, y expresa la posibilidad de que los grupos tomen acuerdos, al margen de los estatutos, ya sea sobre la definición de la línea política del partido, la aprobación de alguna candidatura o algún acercamiento con otros partidos o actores políticos o económicos externos. En ese sentido, dentro de un congreso o consejo nacional o estatal, "entre más grita alguien en el PRD es porque es más débil, los calladitos son los que ya tienen el acuerdo mata estatuto. Y los consejos son eso, es la aplanadora de los grupos que ya están de acuerdo, para los cuales la discusión vale un cacahuate" [sic] (Rosalbina Garavito, entrevista con el autor, 25 de enero de 2010).

Ahora bien, en caso de no lograr un acuerdo por vía informal, en el marco de un proceso de selección al interior del partido, emerge la frase "a navaja limpia" que alude a que, ante la inexistencia de un acuerdo entre grupos en el proceso electoral, se "vale todo", es decir, cualquier práctica como la compra y coacción del voto. Dicha frase "representa la falta de una cultura de diálogo, de respeto a la legalidad y la falta de una cultura de construcción de acuerdos... y es vía de confrontación utilizando métodos, en muchos casos, no legítimos y democráticos” (Jorge Calderón, entrevista con el autor, 07 de enero de 2010).

Así, a diferencia del PAN donde la informalidad puede presentarse en coyunturas críticas, en el PRD es la norma. Por tanto, dichas prácticas informales, conocidas, seguidas, aceptadas y reproducidas generaron certeza a los actores dentro del partido. Siguiendo lo dicho por Helmke y Levitsky (2003), el Sol Azteca brinda evidencia de una lógica competidora, pues las reglas informales (ejemplificadas por la frase “acuerdo, mata estatuto”), suponen la violación sistemática de reglas formales ineficaces. En adición, más que tratarse de prácticas 
que muestran la cordialidad de la competencia, normalmente se trata de prácticas contrarias a la democracia interna.

\section{d) El "gran elector" en el Movimiento Regeneración Nacional}

MORENA es, junto a Partido Encuentro Social, es uno de los partidos más jóvenes, al obtener su registro en 2014. No obstante, la mayoría de los escasos trabajos académicos sobre dicha organización convergen en resaltar el predominio de su líder carismático: Andrés Manuel López Obrador (AMLO) (Espejel-Espinoza, 2015; Navarrete, Camacho y Ceja, 2017; Bolívar, 2017). Ahora bien, dicho predominio se ha materializado gracias a que el líder carismático se transformó en "gran elector", encargado de palomear, presentar y legitimar militantes bajo la figura de "Promotor de la Soberanía Nacional” (PSN). Tiempo después, quienes fueron nombrados PSN se convirtieron en los "candidatos de unidad” de MORENA. Así, "AMLO les daba un espaldarazo al decir: estos son mis elegidos, los otros no tienen ni voz ni voto. Y esa es una decisión poco democrática [sic]” (Gerdoñez, entrevista con el autor, 17 de julio de 2017).

Ahora bien, no se trata de un asunto menor, pues, aunque MORENA es un partido de reciente creación, su votación en entidades como la Ciudad de México, en 2015, y el Estado de México, en 2017, fue sobresaliente. La práctica de nombrar un PNS emergió en 2015, en el contexto de la primera contienda en la que participó MORENA, en el ámbito delegacional de la Ciudad de México, sin embargo, también se manifestó en la designación de los candidatos a gobernador de 2015.

Tabla 2. Promotores y candidatos de MORENA en la Ciudad de México (2015)

\begin{tabular}{|c|c|c|}
\hline Delegación & $\begin{array}{c}\text { Promotor de la Soberanía } \\
\text { Nacional }\end{array}$ & $\begin{array}{l}\text { Coincidencia con el candidato } \\
\text { en } 2015\end{array}$ \\
\hline Álvaro Obregón & Martha Pérez & Sí \\
\hline Azcapotzalco & Pablo Moctezuma & Sí \\
\hline Benito Juárez & Jesús González Schmal & Sí \\
\hline Coyoacán & Bertha Luján & Sí \\
\hline Cuajimalpa & Jenny Saltiel Cohen & Sí \\
\hline Cuauhtémoc & Ricardo Monreal & Sí \\
\hline Gustavo A. Madero & Ramón Jiménez & Sí \\
\hline Iztacalco & Jesús Martín del Campo & Sí \\
\hline Iztapalapa & Clara Brugada & Sí \\
\hline $\begin{array}{l}\text { Magdalena } \\
\text { Contreras }\end{array}$ & Patricia Ortiz & Sí \\
\hline
\end{tabular}




\begin{tabular}{c|c|c|} 
Delegación & $\begin{array}{c}\text { Promotor de la Soberanía } \\
\text { Nacional }\end{array}$ & $\begin{array}{c}\text { Coincidencia con el candidato } \\
\text { en 2015 }\end{array}$ \\
\hline Miguel Hidalgo & José Agustín Ortiz Pinchetti & No: Héctor Vasconcelos \\
\hline Milpa Alta & Guadalupe Chavira & Sí \\
\hline Tláhuac & Rigoberto Salgado & Sí \\
\hline Tlalpan & Claudia Sheinbaum & Sí \\
\hline Venustiano Carranza & Patricia Ruiz & Sí \\
\hline Xochimilco & Avelino Méndez &
\end{tabular}

Fuente: elaboración propia

En el caso de la Ciudad de México, en julio de 2014, AMLO dio aviso, mediante su cuenta de Facebook, a los PSN en el ámbito delegacional (La Jornada, 16 de julio, 2014). A la larga, como se muestra en la Tabla 2, resultó que 15 de los 16 ungidos como PSN serían los candidatos delegacionales de MORENA en la Ciudad de México. En el caso de la Delegación Miguel Hidalgo, el PSN declinó por motivos de salud.

Tabla 3. Promotores y candidatos de MORENA a gubernaturas (2015-2017)

\begin{tabular}{|c|c|c|c|}
\hline Mayo 2014 & Colima & José Francisco Gallardo & Sí \\
\hline Marzo 2014 & Campeche & Layda Sansores & Sí \\
\hline Junio 2014 & Querétaro & Celia Maya & Sí \\
\hline Junio 2014 & San Luis Potosí & Sergio Serrano & Sí \\
\hline Junio 2014 & Guerrero & Lázaro Mazón & $\begin{array}{c}\text { No: Amilcar San- } \\
\text { doval }\end{array}$ \\
\hline Mayo 2014 & Michoacán & María de la Luz Núñez & Sí \\
\hline Junio 2014 & Sonora & Carlos Javier Lamarque & Sí \\
\hline \multirow[t]{2}{*}{ Junio 2014} & $\begin{array}{c}\text { Baja California } \\
\text { Sur }\end{array}$ & Víctor Manuel castro & Sí \\
\hline & & & $\begin{array}{c}\text { Rogelio González } \\
\text { Ramírez }\end{array}$ \\
\hline \multicolumn{4}{|c|}{ Elección para gobernador, Junio 2016} \\
\hline Agosto 2015 & Aguascalientes & Nora Ruvalcaba & Sí \\
\hline Agosto 2015 & Chihuahua & Víctor Quintana & $\begin{array}{c}\text { No: Javier Félix } \\
\text { Muñoz }\end{array}$ \\
\hline Agosto 2015 & Hidalgo & Salvador Torres & Sí \\
\hline
\end{tabular}




\begin{tabular}{c|c|c|c}
\hline Octubre 2015 & Durango & Guillermo Favela & Sí \\
\hline Enero 2016 & Oaxaca & Salomón Jara & Sí \\
\hline Enero 2016 & Puebla & Abraham Quiroz & Sí \\
\hline Septiembre 2015 & Quintana Roo & José Luis Pech & Sí \\
\hline Octubre 2015 & Sinaloa & Jesús Estrada & Sí Sí \\
\hline Septiembre 2015 & Tamaulipas & Héctor Martín Garza & Sín \\
\hline \multicolumn{5}{|c|}{ Elección para gobernador, Junio 2017 } \\
\hline Julio 2016 & Estado de & Delfina Gómez & Sí \\
\hline Agosto 2016 & México & Armando Guadiana & Sí \\
\hline Agosto 2016 & \multicolumn{2}{|c}{ Miguel Ángel Navarro } &
\end{tabular}

Fuente: elaboración propia ${ }^{4}$

Como se puede apreciar en la Tabla 3, en la elección de 2015, siete de los nueve candidatos a gobernador fueron postulados previamente como PSN en su entidad federativa. En algunos casos el nombramiento se realizó con más de un año de antelación al proceso. Los otros dos casos tuvieron que ver con problemáticas aledañas al candidato o la entidad. En Guerrero, debido al lamentable suceso que involucró la desaparición de 43 estudiantes de la Escuela Normal Rural Isidro Burgos en septiembre de 2014, a Lázaro Mazón se le retiró el cargo de PSN, así como la posibilidad de ser candidato. En algunos estados la ausencia del PSN respondió a la falta de cuadros y figuras emblemáticas de MORENA, tal como ocurrió en Nuevo León, donde el dirigente estatal resultó ser el candidato.

Mientras tanto, en 2016, continuó la lógica del PSN, ya que ocho de los nueve candidatos a gobernador adquirieron ese nombramiento antes de la contienda subnacional. Finalmente, la lógica se perpetuó en 2017, pues tres de los candidatos anunciados, un año antes fueron nominados como PSN.

Con lo anterior, queda claro que dicha práctica informal permitió que el partido violara la ley electoral al tener un candidato mucho antes que sus contendientes electorales. Como PSN, los candidatos fueron presentados a la militancia con el aval de su líder moral. Siguiendo a Helmke y Levitsky, MORENA muestra una lógica competidora, pues, a la par de las reglas formales ineficaces operan normas informales más efectivas para nombrar candidatos. Tales mecanismos

4. Cabe aclarar que en las elecciones concurrentes a la presidencial de 2018 se utilizó la figura de Coordinador Territorial en vez de la de Promotor de la Soberanía Nacional. No obstante, la lógica informal siguió siendo la misma. 
informales ilustran el déficit en la democracia interna en MORENA al permitir que candidatos únicos, ya que derivaron en elecciones con candidatos únicos.

\section{Conclusiones}

La lógica informal forma parte ineludible de la realidad partidaria en América Latina (Levitsky y Freidenberg, 2007), sin embargo, es un asunto que no ha cobrado tanta atención frente a los estudios que privilegian la institucionalización formal. Lo anterior significa que la “otra institucionalización”, ámbito de gran relevancia en el análisis político en América Latina, ha sido ignorada. Como se pudo apreciar, la ausencia de informalidad en algunos casos, es señal de posible polarización y escisiones al interior de la organización, como ocurrió en el caso del PAN. Una dinámica distinta es la que tiene lugar en el seno del PRI, donde la lógica informal es síntoma de los arreglos tras bambalinas y lejos del ámbito estatutario.

Otras experiencias como la del PRD, muestra las prácticas informales encarnadas en una realidad fraccionada donde las decisiones, acuerdos y conflictos tienen lugar fuera de los estatutos. Debe señalarse que la informalidad emergió en partidos menos fraccionados, como en el caso de MORENA, dada la prevalencia de un líder carismático. A su vez, la informalidad se configuró en un mecanismo que le ha permitido al partido tener cierta ventaja frente a sus adversarios, al designar a los posibles candidatos de manera anticipada.

El estudio de la informalidad partidista es un área fértil de investigación. Sin embargo, este primer acercamiento únicamente puso atención en el papel que juega la informalidad en la configuración organizacional de los partidos políticos en México. En ese sentido, la contribución del trabajo estriba en brindar evidencia empírica sobre cómo la informalidad aporta el sello característico a las organizaciones partidistas, asunto usualmente ignorado por la literatura existente.

Por consiguiente, es necesario continuar indagando para aportar evidencia empírica sobre la informalidad partidaria, particularmente sobre su función en contextos específicos, así como sus consecuencias.

En esta investigación se concluye que el peso de la informalidad difiere entre los casos presentados. Así, mientras que en el PRD ha sido una constante en la vida organizativa, en MORENA, la informalidad parece ocupar un lugar cada vez más preponderante en la toma de decisiones. Aunque en el primer caso se expresa en la existencia de un cúmulo de grupos internos, en el segundo, la informalidad se manifiesta en la presencia de un líder carismático que decide sobre las preferencias de la militancia. Entre tanto, en una época distinta, durante el presidencialismo mexicano, el PRI fue un claro ejemplo de un partido subordina- 
do al titular del Poder Ejecutivo. A pesar de esas particularidades, en los tres casos se trata de instituciones informales competitivas cuyo cumplimiento supone la violación de reglas formales ineficaces donde la militancia queda marginada.

En adición, Acción Nacional ha sido la organización donde la informalidad sólo se ha presentado en ciertas coyunturas, sin embargo, es un indicador eficaz a la hora de identificar el nivel del conflicto interno. En este partido, la informalidad es una institución complementaria, pues permitió mejorar el desempeño de las reglas formales. Sin embargo, la militancia también es marginada en la toma de decisiones relevantes. Adicionalmente, vale la pena resaltar que la informalidad muestra la flexibilidad organizativa que no siempre se traduce en concentración de poder, como sucede en los tres casos anteriores. La informalidad puede mostrar la fragmentación de poder a manos de los grupos internos (como ocurre con el PRD), o bien, tiende a concentrarse en el Presidente en funciones (PRI), o en el líder carismático en turno (MORENA). En ese sentido, vale la pena explorar, en futuras investigaciones, cómo la informalidad no siempre se traduce en el deterioro de la democracia interna. A su vez, es necesario identificar el papel que tiene la informalidad en otro tipo de partidos como los minoritarios (Partido del Trabajo, Partido Verde Ecologista de México, Movimiento Ciudadano, Partido Nueva Alianza o Partido Encuentro Social).

Incluso, resulta valioso explorar la diversidad (Ragin, 2007) con otras realidades que permitan apreciar las múltiples manifestaciones y funciones de la informalidad. En ese sentido, se podría comparar algunos de los partidos aquí analizados con sus contrapartes en América Latina. Por ejemplo, el caso del PRD con el Frente País Solidario, debido a que ambos son partidos fraccionados; o bien, el PRI con el Partido Justicialista, organizaciones que mantuvieron una posición dominante durante un largo periodo.

Finalmente, es preciso avanzar en el estudio de la informalidad partidaria. Analizar este tipo de fenómenos políticos acerca a la comprensión de la realidad partidaria, gracias a lo cual será posible plantear hipótesis de carácter causal, así como comparaciones que permitan dar cuenta de variaciones y similitudes. Con ello, avanzaría el conocimiento sobre las dinámicas organizativas de los partidos.

\section{Referencias}

Araujo, K. (2009a). El ordinario trabajo moral del sujeto. En K. Araujo (Coord.). ¿Se acata pero no se cumple? Estudio sobre las normas en América Latina (pp. 91-118). Santiago de Chile: LOM Editores. 
Araujo, K. (2009b). Introducción. En K. Araujo (Coord.). ¿ Se acata pero no se cumple? Estudio sobre las normas en América Latina (pp. 7-18). Santiago de Chile: LOM Editores.

Bolívar, R. (2017). Movimiento Regeneración Nacional: democracia interna y tendencias oligárquicas. Foro Internacional, 228, 460-489. http:/ /www.scielo.org.mx/pdf/fi/v57n2/0185-013X-fi-57-02-00460.pdf [Consultado el 11 de abril de 2019].

Carpizo, J. (1987). El presidencialismo mexicano. México, D. F: Siglo XXI Editores.

Corona, G. (2003). La selección de los dirigentes nacionales del PRI. En F. Reveles (Coord.). PRI: crisis y refundación (pp. 153-220). México, D. F: Gernika.

Duque, J. (2005). La institucionalización partidista. Una propuesta de abordaje de las estructuras organizativas partidistas. Estudios Políticos, 27, 103-127. https://www.redalyc.org/pdf/164/16429055006.pdf [Consultado el 11 de abril de 2019].

Espejel-Espinoza, A. (2010). "Ni buenos, ni malos": Juego sucio y resultados en los procesos de elección de dirigencia nacional en el Partido de la Revolución Democrática (1999-2008) (Tesis de Maestría, inédita). México: FLACSO

Espejel-Espinoza, A. (2015). Orígenes organizativos y derroteros estatutarios del Movimiento de Regeneración Nacional y el Partido de la Revolución Democrática. Entre carisma y grupos políticos. Estudios Políticos, 35, 11-38.

http://www.revistas.unam.mx/index.php/rep/article/view/49371/44411 [Consultado el 13 de mayo de 2019].

Espejel-Espinoza, A. (2016). Origen y cambia la concentración y intrapartidaria de poder. Los casos del PRI, PAN y PRD. Intersticios Sociales, 1(12), 1-35. http://www.scielo.org.mx/pdf/ins/n12/2007-4964-ins-12-00009.pdf Consultado el 13 de mayo de 2019].

Girola, L. (2009). La cultura del como sí. Normas, anomia transgresión en la sociedad mexicana. En K. Araujo (Coord.). ¿Se acata pero no se cumple? Estudio sobre las normas en América Latina (pp. 19-56). Santiago de Chile: LOM Editores. 
González, A. M.; Gómez, A. (2010). Una amistad sin sombras. Correspondencia entre Manuel Gómez Morín y Efraín González Luna. 1934-1964. México, D. F: Fondo de Cultura Económica.

Helmke, G.; Levitsky, S. (2003). Informal Institutions and Comparative Politics: A research agenda. In Helmke, G. \& Levitsky, S. (Eds.). Informal Institutions and Politics in Latin America (pp. 1-33). Estados Unidos de Norteamérica: Kellog Institute for International Studies.

Hernández, R. (2008). El centro dividido. La nueva autonomía de los gobernadores. México, D. F.: El Colegio de México.

Huntington, S. (1968). Political Order in Changing Societies. Connecticut, Estados Unidos: Yale University Press.

Levitsky, S. (2003). Los partidos obreros en transformación en América Latina: El Partido Justicialista Argentino en perspectiva comparada. En M. Alcántara y E. Barahona (Coords.). Política, dinero e institucionalización partidista en América Latina (pp. 245-302). México. Universidad Iberoamericana.

Levitsky, S.; Freidenberg, F. (2007). Organización informal de los partidos en América Latina. Desarrollo Económico, 46(184), 539-568. https://www.researchgate.net/publication/263697026_Organizacion_Informal_de_los_ Partidos_en_America_Latina [Consultado el 23 de mayo de 2019].

Martínez, V. H. (2005). Fisiones y fusiones, divorcios y reconciliaciones. La dirigencia del Partido de la Revolución Democrática. México: Plaza y Valdés.

Michels, R. (1983). Los partidos políticos. Un estudio sociológico de las tendencias oligárquicas de la democracia interna. Buenos Aires, Argentina: Amorrortu.

Navarrete, J.; Camacho, O.; Ceja M. (2017). Formación, liderazgo y desempeño electoral de MORENA. Revista Mexicana de Estudios Electorales, 1(18), 11-60. http://dcsh.izt.uam.mx/cen_doc/cede/imgsSOMEE/SOMEE_2017_2/ Formaci\%C3\%B3n,\%2oliderazgo\%20y\%20desempe\%C3\%B 10\%20electoral\%20de\%20Morena.pdf [Consultado el 12 de abril de 2019].

O’Donell, G. (1997). Contrapuntos: ensayos escogidos sobre autoritarismo y democratización. Buenos Aires, Argentina: Paidós. 
Ortiz, P. (2008). Partidos políticos en Guatemala: entre institucionalidad y pragmatismo político. Ciudad de Guatemala: FLACSO.

Ostrogorski, M. (2008). La democracia y los partidos políticos. Barcelona, España: Trotta.

Panebianco, A. (1990). Modelos de partido. Madrid, España: Alianza Universitaria.

Partido Revolucionario Institucional, PRI. (2003). Esbozo Histórico. México, D. F.: Partido Revolucionario Institucional.

Patton, M. (2002). Qualitative Research and Evaluation Methods. California, Estados Unidos: Sage.

Pedrosa, F. (2005). Las relaciones personales también importan. Instituciones informales, redes y partidos políticos. Revista Hispana para el Análisis de Redes Sociales, 1(2), 1-14.

http://revista-redes.rediris.es/webredes/arsrosario/o2-Pedrosa.pdf [Consultado el 12 de abril de 2019].

Ragin, C. (2007). La construcción de la investigación social. California, Estados Unidos: Sage.

Randall, V.; Svásand, L. (2002). Party Institutionalization in New Democracies. Party Politics, 8(1), 5-29.

https://journals.sagepub.com/doi/10.1177/1354068802008001001 [Consultado el 23 de abril de 2019].

Reveles, F. (2002). PAN: Los signos de la institucionalización. México, D. F.: Gernika.

Reveles, F. (2003). Partido Revolucionario Institucional: Crisis y refundación. México, D. F.: Gernika.

Reveles, F. (2004). Partido de la Revolución Democrática. Los problemas de institucionalización. México, D. F.: Gernika. 